\title{
BMJ Open Early indicators of disease progression in Fabry disease that may indicate the need for disease-specific treatment initiation: findings from the opinion- based PREDICT-FD modified Delphi consensus initiative
}

Derralynn A Hughes, ${ }^{1,2}$ Patricio Aguiar, ${ }^{3,4}$ Patrick B Deegan, ${ }^{5,6}$ Fatih Ezgu, ${ }^{7}$ Andrea Frustaci (D) , Olivier Lidove, ${ }^{9}$ Aleš Linhart, ${ }^{10}$ Jean-Claude Lubanda, ${ }^{10}$ James C Moon, ${ }^{11}$ Kathleen Nicholls, ${ }^{12,13}$ Dau-Ming Niu, ${ }^{14,15}$ Albina Nowak, ${ }^{16,17}$ Uma Ramaswami, ${ }^{1}$ Ricardo Reisin, ${ }^{18}$ Paula Rozenfeld, ${ }^{19}$ Raphael Schiffmann, ${ }^{20}$ Einar Svarstad, ${ }^{21,22}$ Mark Thomas, ${ }^{23}$ Roser Torra, ${ }^{24}$ Bojan Vujkovac, ${ }^{25}$ David G Warnock, ${ }^{26}$ Michael L West, ${ }^{27}$ Jack Johnson, ${ }^{28,29}$ Mark J Rolfe (1) ,30 Sandro Feriozzi ${ }^{31}$

To cite: Hughes DA, Aguiar P, Deegan PB, et al. Early indicators of disease progression in Fabry disease that may indicate the need for disease-specific treatment initiation: findings from the opinion-based PREDICT-FD modified Delphi consensus initiative. BMJ Open 2020;10:e035182. doi:10.1136/ bmjopen-2019-035182

- Prepublication history and additional material for this paper are available online. To view these files, please visit the journal online (http://dx.doi. org/10.1136/bmjopen-2019035182).

Received 21 October 2019 Revised 19 June 2020 Accepted 16 July 2020

Check for updates

(c) Author(s) (or their employer(s)) 2020. Re-use permitted under CC BY-NC. No commercial re-use. See rights and permissions. Published by BMJ.

For numbered affiliations see end of article.

Correspondence to

Professor Derralynn A Hughes; rmgvdah@ucl.ac.uk

\section{ABSTRACT}

Objectives The PRoposing Early Disease Indicators for Clinical Tracking in Fabry Disease (PREDICT-FD) initiative aimed to reach consensus among a panel of global experts on early indicators of disease progression that may justify FD-specific treatment initiation.

Design and setting Anonymous feedback from panellists via online questionnaires was analysed using a modified Delphi consensus technique. Questionnaires and data were managed by an independent administrator directed by two non-voting cochairs. First, possible early indicators of renal, cardiac and central/peripheral nervous system (CNS/PNS) damage, and other disease and patientreported indicators assessable in routine clinical practice were compiled by the cochairs and administrator from panellists' free-text responses. Second, the panel scored indicators for importance (5-point scale: $1=$ not important; $5=$ extremely important); indicators scoring $\geq 3$ among $>75 \%$ of panellists were then rated for agreement (5-point scale: 1 =strongly disagree; $5=$ strongly agree). Indicators awarded an agreement score $\geq 4$ by $>67 \%$ of panellists achieved consensus. Finally, any panel-proposed refinements to consensus indicator definitions were adopted if $>75 \%$ of panellists agreed.

Results A panel of 21 expert clinicians from 15 countries provided information from which 83 possible current indicators of damage (kidney, 15; cardiac, 15; CNS/PNS, 13; other, 16; patient reported, 24) were compiled. Of 45 indicators meeting the importance criteria, consensus was reached for 29 and consolidated as 27 indicators (kidney, 6; cardiac, 10; CNS/PNS, 2; other, 6; patient reported, 3) including: (kidney) elevated albumin:creatinine ratio, histological damage, microalbuminuria; (cardiac) markers of early systolic/diastolic dysfunction, elevated serum cardiac troponin; (CNS/PNS) neuropathic pain,
Strengths and limitations of this study

- A globally representative panel of clinician-experts in Fabry disease (FD) was recruited.

- Group interaction bias was minimised by the anonymous consensus process.

- The response rate was $>95 \%$ at each round of the consensus process.

- Scoring of FD indicators reflects the real-world views of clinicians.

gastrointestinal symptoms suggestive of gastrointestinal neuropathy; (other) pain in extremities/neuropathy, angiokeratoma; (patient-reported) febrile crises, progression of symptoms/signs. Panellists revised and approved proposed chronologies of when the consensus indicators manifest. The panel response rate was $>95 \%$ at all stages.

Conclusions PREDICT-FD captured global opinion regarding current clinical indicators that could prompt FD-specific treatment initiation earlier than is currently practised.

\section{INTRODUCTION}

Fabry disease (FD) affects individuals deficient in lysosomal alpha-galactosidase A. The disease is X-linked, with an estimated prevalence of up to 1 in 40000 , and its multisystem pathology is caused by intracellular accumulation of globotriaosylceramide $(\mathrm{Gb} 3){ }^{1}$ FD presents with highly variable symptomatology ranging from patients who are asymptomatic to those severely affected with multiorgan 
damage. ${ }^{1}$ The rate at which FD progresses also varies considerably. ${ }^{1}$ This poses a major challenge for physicians in determining prognosis, and consequently a diagnosis of FD does not automatically merit initiation of FD-specific treatment with enzyme replacement therapy (ERT) or chaperone therapy. Instead, physicians must monitor patients regularly to identify signs that may warrant treatment initiation. The decision whether to treat may be complicated by the high costs of FD-specific treatments ${ }^{2}$ and by the considerable patient burden associated with hospital treatment if home therapy is unavailable or inappropriate. $^{34}$

In 2015, the European Fabry Working Group (EFWG) published consensus criteria for initiation and withdrawal of ERT in patients with FD. ${ }^{1}$ The general recommendation applied to classically affected males and females and to non-classically affected males, and was to initiate treatment when clinical signs of kidney, heart or central nervous system (CNS) involvement, pain or gastrointestinal symptoms first appeared. ${ }^{1}$ Treatment of classically affected males aged $\leq 16$ years could also be considered in the absence of signs or symptoms of organ involvement, as could treatment of non-classically affected females with early clinical signs attributed to FD. ${ }^{1}$ Initiation or continuation of FD-specific treatment was to be considered on an individual basis, and certain recommendations were made to withhold treatment (eg, in patients with endstage renal disease with no option for renal transplant and advanced heart failure, or in patients with severe cognitive decline). ${ }^{1}$

The EFWG guidelines provide a valuable framework for clinical decision making in FD, but important recent advances in the field suggest that revising these recommendations may now be appropriate. An increasing body of evidence supports the early initiation of ERT in patients with $\mathrm{FD},{ }^{5-8}$ and several studies show that the best outcomes of ERT are in patients with the least organ damage at treatment initiation. ${ }^{56-12}$ A study comparing response to FD-specific treatment after 1 year among treatment-naive men starting ERT before the age of 25 years with that among men who started treatment later, found a significantly greater reduction in plasma levels of globotriaosylsphingosine (lyso-Gb3; a marker of disease severity in FD) in the group treated early. ${ }^{13}$

As well as new clinical outcome data, new imaging techniques such as cardiac MRI (cMRI) ${ }^{14}$ and ${ }^{123}$ I-metaiodobenzylguanidine single-photon emission $\mathrm{CT}^{15}$ will likely offer the means to detect very early FD-related organ damage not identified by traditional assessment methods. Such approaches facilitate FD-specific treatment initiation before more advanced signs appear and irreversible organ damage occurs.

We conducted the international PRoposing Early Disease Indicators for Clinical Tracking in Fabry Disease (PREDICT-FD) modified Delphi initiative to establish expert consensus on early clinical indicators that may prompt when FD-specific treatment should be initiated in treatment-naive patients. The Delphi process is a widely used, validated technique for developing expert consensus when evidence is limited and has generated simple, robust clinical guidance, including for the diagnosis and management of patients with FD. ${ }^{16-18}$ The stepwise use of questionnaires and the maintenance of anonymity of the experts consulted minimises data distortion that can arise from the pressure on individuals within a group to conform to a dominant view. ${ }^{19}$ As well as examining the most relevant early clinical indicators of FD progression, we also aimed to gain agreement on when to initiate and to stop FD-specific treatment in different patient groups in different scenarios. The intention is that these findings will raise awareness among specialist and general physicians of the early clinical cues that should prompt consideration of disease-specific treatment initiation in patients with FD, so that disease progression and irreversible organ damage in these patients is minimised or avoided.

\section{METHODS}

The modified Delphi process used in PREDICT-FD is described below and summarised in figure 1 .

\section{Selection of chairs and expert panel}

Two leading global experts in FD were invited to be non-voting cochairs of the PREDICT-FD initiative. The cochairs selected an international group of FD experts to form the voting panel. Panel members were nominated based on track record and demonstrated expertise in the field, according to factors such as research activities, participation in national or regional FD management initiatives and authorship of relevant peer-reviewed publications. Nominated panellists were recruited on behalf of the initiative cochairs by an independent third-party administrator (Oxford PharmaGenesis, Oxford, UK).

\section{Modified Delphi process}

Under the direction of the PREDICT-FD cochairs, the third-party administrator drafted a study protocol, which was reviewed and approved by both cochairs and by a patient representative before commencement of the initiative. A non-exhaustive literature search was also conducted by the administrator for the cochairs and was used to inform aspects of the initiative (see online supplementary appendix). All stages of the initiative, including content development, data collation, data processing and reporting, were overseen by the cochairs and conducted by the independent third-party administrator. Expert panel responses were gathered anonymously via an online survey platform (SurveyMonkey, SurveyMonkey Europe, Dublin, Ireland). For tracking purposes, the administrator knew the identities of responding panellists, but no identifying information was shared with the cochairs or other panel members. Panellists remained anonymous to each other throughout the Delphi stages. Circulation of the questionnaires, and collection and processing of the panel's responses was conducted between January and 


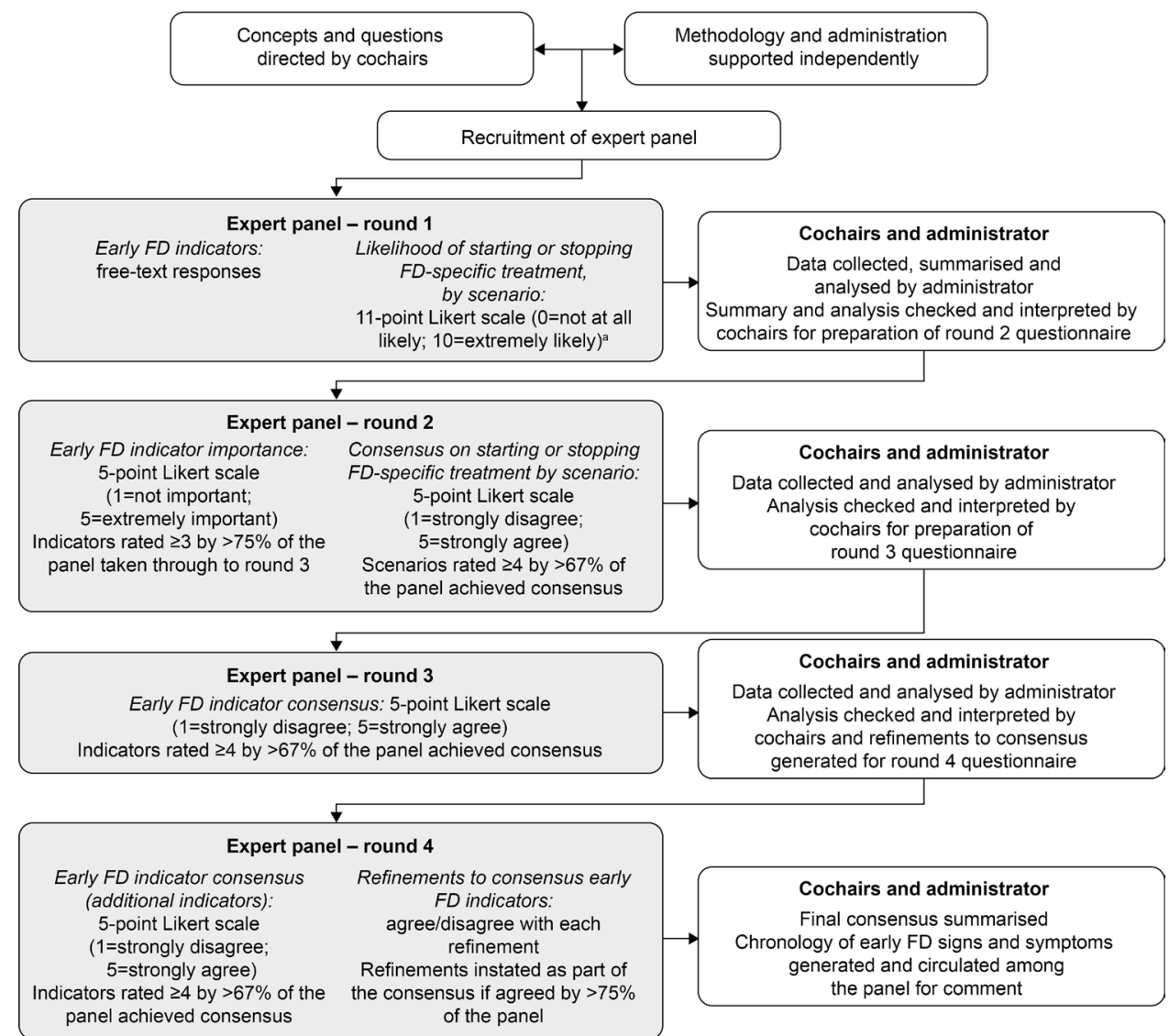

Figure 1 PREDICT-FD modified Delphi consensus methodology. ${ }^{\mathrm{a}} \mathrm{A}$ threshold median likelihood score of 7.5 was set a priori. For questions about the likelihood of initiating treatment, agreement for initiation was sought in round 2 if a scenario was awarded a median score of $\geq 7.5$ and agreement not to initiate treatment sought if the score was $<7.5$. Similarly, for questions about cessation of treatment, agreement to stop treatment was sought in round 2 if a scenario was awarded a median score $\geq 7.5$ and agreement not to stop treatment sought if the score was $<7.5$. PREDICT-FD, PRoposing Early Disease Indicators for Clinical Tracking in Fabry Disease.

September 2018. Except for comment fields included in the questionnaires, all questions were compulsory. No controlled feedback was provided to panellists between rounds.

Further details on the design of the modified Delphi initiative, including all questionnaires, are provided in the online supplementary appendix. Achieving consensus with three rounds of questionnaires was planned. In round 1, information was solicited regarding panellists' FD clinical practices, number of years spent treating patients with FD and number of patients with FD typically managed in their practices. Panellists provided free-text responses to open questions soliciting suggestions for early indicators of renal, cardiac and CNS damage that can be assessed in current routine clinical practice, or that are not assessed routinely at present, but might be in the future. Additional round 1 questions explored symptoms experienced by patients with FD that could contribute to initiating FD-specific treatment. Attitudes towards FD-specific treatment initiation or cessation were also investigated by asking panellists to rate on an 11-point scale ( $0=$ not at all likely; $10=$ extremely likely) the likelihood that they would start or stop FD-specific treatment in different patient groups and clinical scenarios proposed by the cochairs.

Among questions in round 1 that solicited free-text responses, the administrator identified similar themes among the responses and created provisional groupings for review by the cochairs. The cochairs checked and revised the groupings to exclude indicators that are not widely used, are known to be of greater relevance in latestage than in early-stage disease or are poorly indicative of FD status and progression. The administrator generated lists of indicators and compiled responses from the panel regarding attitudes to FD-specific treatment initiation or cessation in different patient groups, determining the panel's median likelihood scores for starting or stopping FD-specific treatment.

In round 2, panellists rated the importance of each indicator on a 5-point Likert scale (1=not important; $2=$ slightly important; $3=$ important; $4=$ very important; $5=$ extremely important). Regarding scenarios for initiation or cessation of FD-specific treatment, if a scenario was awarded a median likelihood score of $\geq 7.5$ in round 1, agreement was sought whether to start or 
to stop FD-specific treatment. In contrast, if the score was $<7.5$, agreement was sought whether to start or to stop treatment. Panellists rated their level of agreement using a 5-point Likert scale ( $1=$ strongly disagree; $2=$ disagree; $3=$ neither agree nor disagree; $4=$ agree; $5=$ strongly agree). Importance and agreement ratings were compiled by the administrator. It was specified a priori that indicators awarded an importance score of $\geq 3$ by $>75 \%$ of the panel would be tested for consensus in round 3, and that agreement on treatment recommendations would be reached if an agreement score of $\geq 4$ was awarded by $>67 \%$ of the panel. All ratings compiled by the administrator were reviewed by the cochairs as per the predefined scores and consistent with previous Delphi initiatives ${ }^{20}{ }^{21}$; agreement on treatment recommendations concluded in round 2 . In round 3, panellists rated their level of agreement with each indicator that had met the designated importance criteria in round 2, using the 5-point Likert scale already described. Consensus was established using the same a priori criteria already described. Agreement scores were compiled by the administrator and reviewed by the cochairs.

Round 4 was included post hoc to capture the panel's level of agreement with certain indicators that met the importance criteria in round 2 but which were inadvertently omitted from round 3. Panel members were also asked whether they agreed or disagreed with refinements proposed for several indicators that achieved consensus in round 3 and these were adopted if $>75 \%$ of the panel agreed; refinements were informed by comments made by panel members during the first three rounds. Panellists' responses were compiled by the administrator, reviewed by the cochairs, and any new consensus terms combined with those identified in round 3 .

\section{Chronology of signs and symptoms}

After generating the refined list of consensus indicators, timelines were developed under the direction of the cochairs showing when each indicator typically manifests during the disease course in relation to established indicators currently recommended as triggers for treatment initiation. Indicators manifesting before and after established indicators were termed 'early' and 'late', respectively. Indicators featuring in the chronologies were grouped as renal, cardiac or patient reported/ other. The cochairs agreed a draft chronology for each group, and these proposals were submitted to each panel member for comment and amendment. Panel responses were collated, and the chronologies revised by the administrator then approved by the cochairs. The chronologies were developed between December 2018 and January 2019; Delphi consensus techniques were not applied to this part of the initiative.

\section{Statistical analyses}

The study was exploratory; no hypotheses were tested and only descriptive statistical analyses were performed.

\section{Patient and public involvement statement}

A leadership representative from the Fabry International Network (FIN), JJ, was invited to participate in the project in a non-voting role. The representative reviewed and approved the initial protocol and round 1 questionnaire, and facilitated the involvement of three patients with FD (one from the USA and two from outside the USA) in reviewing these materials. This ensured that any appropriate feedback from the patients could be incorporated into materials before distributing the round 1 questionnaire. Additional roles of the FIN representative included capturing these patients' views on the outcomes of the initiative, and reviewing and approving the final study report.

\section{RESULTS \\ PREDICT-FD expert panel demographics and clinical experience}

In total, 23 experts were invited to join the expert panel; one declined to participate, and one did not complete round 1 and was excluded from the analysis. Thus, the panel comprised 21 physicians representing 15 countries (Argentina, Australia, Canada, Czech Republic, France, Italy, Norway, Portugal, Slovenia, Spain, Switzerland, Taiwan, Turkey, UK, USA). All panellists had managed male and female patients with FD; most panellists had experience of managing both patients with classical and those with non-classical FD (table 1).

The majority of panellists $(18(85.7 \%))$ practised in public teaching hospitals. Panellists had treated patients with FD for a mean of 15.5 years and four panellists $(19.0 \%)$ had $>20$ years of clinical experience with FD. Specialties most commonly represented were nephrology $(8(38.1 \%))$, metabolic diseases $(5(23.8 \%)$, of whom 3 $(14.3 \%)$ also specialised in genetics) and cardiology (4 $(19.0 \%))$; haematology, immunology, neurology, paediatrics, internal medicine, biochemistry and angiology were also represented. Overall, the panel managed an estimated 2079 patients, $40.7 \%$ of whom were male; $64.5 \%$ of patients had classical FD (table 1). A response rate of $95.5 \%(21 / 22)$ was achieved during round 1 of the modified Delphi process; thereafter all 21 panellists responded.

\section{Consensus on current and potential future indicators of disease progression in FD}

Indicators achieving consensus in round 3 of the modified Delphi process were further refined in round 4 (see section 'Refinements to consensus indicators' for further information); the final list of consensus indicators is summarised in table 2 . Results by organ system and category are described below.

\section{Indicators of renal damage}

Following consolidation by the cochairs, 15 indicators of early renal damage in current use and 19 potential future indicators were collated from round 1. Of these, seven 
Table 1 PREDICT-FD modified Delphi expert panel clinical experience

\begin{tabular}{|c|c|}
\hline Clinical experience $(n=21)$ & \\
\hline \multicolumn{2}{|l|}{ Main clinical practice* } \\
\hline Private teaching hospital & $1(4.8)$ \\
\hline Private hospital & 0 \\
\hline Public teaching hospital & $18(87.5)$ \\
\hline Public non-teaching hospital & 0 \\
\hline Research centre & $6(28.6)$ \\
\hline \multicolumn{2}{|c|}{ Duration of FD clinical experience, years } \\
\hline Mean (SD) & $15.5(7.5)$ \\
\hline $0-10$ & $6(28.6)$ \\
\hline $11-20$ & $11(52.4)$ \\
\hline $21-30$ & $4(19.0)$ \\
\hline
\end{tabular}

\section{Number of patients with FD managed}

$\begin{array}{lr}\text { Mean }(\mathrm{SD}) & 99(81) \\ 1-50 & 4(19.0) \\ 51-100 & 12(57.1) \\ 101-200 & 3(14.3) \\ >200 & 2(9.5)\end{array}$

\section{Patient summary†}

\begin{tabular}{lr} 
Male & $847(40.7)$ \\
Female & $1232(59.3)$ \\
Classical FD & $1341(64.5)$ \\
Non-classical FD & $738(35.5)$ \\
\hline
\end{tabular}

Data are shown as number (\%) of respondents unless otherwise stated.

${ }^{*}$ Respondents could select more than one option.

†Patient $\mathrm{n}(\%)$ values are estimates, derived from total patient numbers and estimated sex and FD-type breakdown reported by each panellist.

FD, Fabry disease; PREDICT-FD, PRoposing Early Disease Indicators for Clinical Tracking in Fabry Disease.

current and two future indicators met the predefined importance criteria in round 2. Consensus was reached for the following current indicators (see online supplementary table S1): elevated urine albumin:creatinine ratio; histological damage (lesions associated with Gb3 deposition); microalbuminuria; abnormal glomerular filtration rate (GFR); decline in iohexol GFR and podocyte inclusions in renal biopsies. Consensus was not achieved for any future indicators.

\section{Indicators of cardiac damage}

After consolidation at the end of round 1, 15 current and 14 future indicators of early cardiac damage were identified, and 12 current and 3 future indicators met the importance criteria in round 2. Consensus was reached for 10 current indicators, 3 of which also reached consensus as future indicators (see online supplementary table S2). The indicators deemed important, both currently and in the future, were: reduced myocardial $\mathrm{T} 1$ relaxation time on cMRI; elevated serum cardiac troponin; and elevated serum N-terminal probrain natriuretic peptide (NT-pro-BNP). The other important current indicators were: markers of early systolic/diastolic dysfunction; early indicators of left ventricular hypertrophy (LVH); histological damage (lesions associated with Gb3 deposition) in endomyocardial biopsies; late gadolinium enhancement on cMRI; abnormal ECG; abnormal echocardiogram; and, specifically, abnormal wall motion revealed by echocardiogram.

\section{Indicators of peripheral nervous system damage}

In round 1 following consolidation, 13 current and 13 future indicators were identified, with 5 and 2 indicators, respectively, subsequently meeting the importance criteria in round 2 (see online supplementary table S3). Consensus was reached for neuropathic pain and gastrointestinal symptoms suggestive of gastrointestinal neuropathy as current indicators; no consensus was achieved for future indicators.

\section{Other indicators}

When asked for further information about early indicators of FD, such as non-organ-specific symptoms, consensus was reached for five indicators (see online supplementary table S4): pain in extremities/neuropathy; angiokeratoma; organ biopsy (including skin biopsy for small-fibre neuropathy); gastrointestinal symptoms (including bloating, pain, diarrhoea/frequent diarrhoea or constipation); and sweating abnormalities or heat/ exercise intolerance.

\section{Patient-reported indicators}

Panellists were asked to list what they considered to be the earliest signs and symptoms relevant to FD progression and FD-specific treatment initiation, and also to list patient-reported signs and symptoms relevant to FD-specific treatment initiation. When the responses were combined, consensus was achieved for the following six patient-reported indicators: stroke/transient ischaemic attack; febrile crises; patient-reported progression of symptoms/signs of FD (such as acral burning paraesthesias, heat intolerance, impaired sweating, fatigue, depression, pain, gastrointestinal symptoms, shortness of breath, palpitations, peripheral oedemas); diarrhoea/ frequent diarrhoea; angiokeratoma; and neuro-otological abnormalities (see online supplementary table S5). Based on consensus reached in round 4, stroke/transient ischaemic attack and diarrhoea/frequent diarrhoea were reclassified among 'other indicators', and neurootological abnormalities was discarded (see 'Refinements to consensus indicators').

\section{Indicators under research}

Of the eight indicators that were the focus of experimental studies or ongoing research, five were deemed important, and two achieved consensus (see online supplementary table $\mathrm{S6}$ ): reduced quality of life and high gastrointestinal symptom scores. 

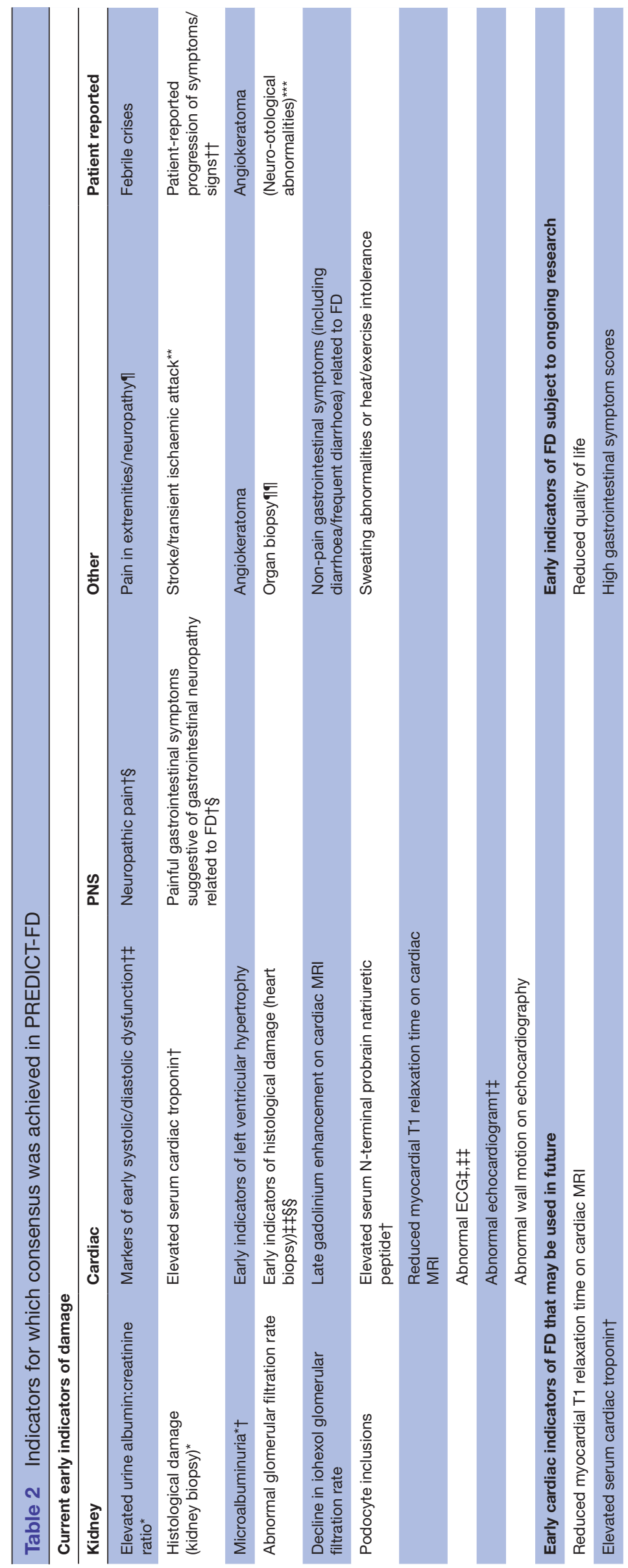

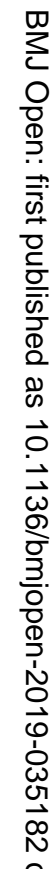

옥

$\vec{\circ}$

웅

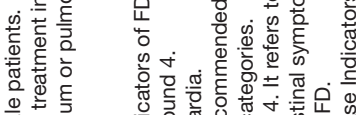

区

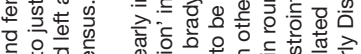

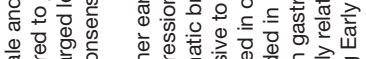

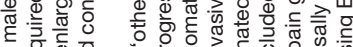

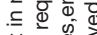

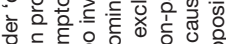

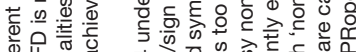

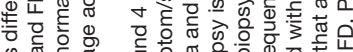

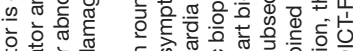

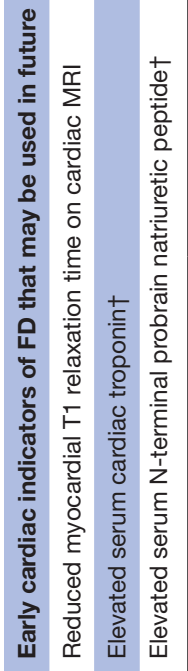

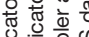

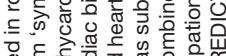

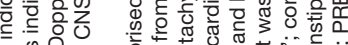

曹舫

定

o

\&

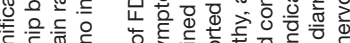

क⿹ 口⿱

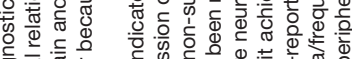

o

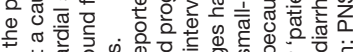

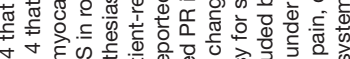

寸

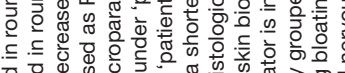

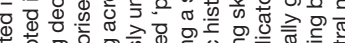

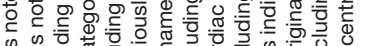

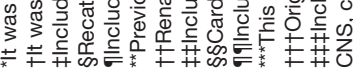

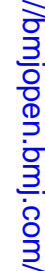

인

긍

No

กิ

श्र

$\stackrel{0}{\mathbb{D}}$

$\stackrel{+}{+}$

$\stackrel{\frac{0}{0}}{\frac{0}{D}}$

胥

$\frac{0}{2}$

음 
Refinements to consensus indicators

During the first three rounds, panellists offered additional information about the indicators, typically to define broad indicators more precisely. Comments on the current indicators that achieved consensus were reviewed by the cochairs, and proposed clarification on 23 of these was circulated to the panel in round 4, either to endorse new information or to provide an opportunity to include additional information. The panel reached agreement on refinements to 19 of these indicators (see online supplementary table S7; 'neuro-otological abnormalities' was excluded from the consensus because it encompassed the other indicators 'vertigo', 'hearing loss' and 'tinnitus' that had not achieved consensus (see online supplementary tables S4,S5). The current and potential future indicators, as well as those under research, that achieved final consensus are summarised in table 2; explanatory table footnotes describe the refinements made in round 4 based on feedback from the panel.

\section{Chronology of manifestation of indicators during the disease course}

Indicators that achieved consensus were allocated to three groups: renal; cardiac; and patient reported/ other, and a chronology was developed for each group (figure 2A-C).

\section{Initiation and cessation of FD-specific treatment in patients with FD}

In round 1, the panel rated the likelihood of initiating FD-specific treatment in different scenarios (patients asymptomatic for organ damage, symptomatic patients not meeting guideline criteria, patients meeting guideline criteria) in five different patient groups (defined by sex, age group, and classical or non-classical FD) (see online supplementary figure S1A). The panel's level of agreement in round 2 with proposals that treatment should or should not be started in different patient groups in different scenarios is summarised in table 3. Agreement was reached in round 2 that FD-specific treatment should be initiated in all males aged $\geq 16$ years with classical disease, and in males of any age with classical disease and with early indicators of organ damage, irrespective of whether these symptoms meet the EFWG recommendations for treatment initiation. ${ }^{1}$ Agreement that FD-specific treatment should be initiated was also reached for all female patients and for male patients with non-classical disease with indicators meeting the EFWG guideline criteria. ${ }^{1}$ Agreement not to start treatment was reached only for asymptomatic females with non-classical FD (table 3). However, when asked if all patients who meet the EFWG guideline criteria ${ }^{1}$ should receive FD-specific treatment, the panel did not reach agreement (mean (median) score, 3.4 (4); score $\geq 4,11(52.4 \%)$ ), including for female patients with classical FD and male patients with non-classical FD.

The panel's responses regarding starting or stopping FD-specific treatment in scenarios relating to organ damage are summarised in table 4 and online supplementary figure S1B. Agreement was reached that treatment should be initiated in patients with evidence of damage to a single organ system, irrespective of whether that organ system was being treated by a non-Fabry-specific intervention (eg, renal replacement therapy, kidney transplant or cardiac pacemaker, etc), and that FD-specific treatment of such patients should not be stopped, were such a therapy to become necessary. Agreement was also reached that FD-specific treatment should be initiated and should not be stopped in patients receiving separate therapies for damage to multiple organ systems (such as a combination of renal replacement therapy, kidney transplant and/or cardiac pacemaker, etc). The group in which the panel was least likely to initiate or to stop FD-specific treatment was that comprising patients who were receiving no separate therapy for multiple organ system damage. However, no agreement was reached for either scenario. The panel also did not reach agreement on the question of whether all patients with FD should remain on disease-specific treatment, irrespective of organ damage or any related treatment (mean (median) agreement score, 2.2 (2); agreement score $\geq 4,6(28.6 \%)$ ).

\section{DISCUSSION}

The PREDICT-FD panel was convened to identify early clinical indicators that could prompt disease-specific treatment initiation in patients with FD, thereby minimising disease progression. The panel reached consensus on 27 early renal, cardiac, peripheral nervous system (PNS), patient-reported and other indicators of disease progression that can currently be assessed in FD clinics (table 2). Other indicators that were considered important but where no consensus was reached or that were categorised as being of no importance, are summarised in the supplementary tables. Three indicators of cardiac damage were also identified that might be adopted more widely for routine use in future and the utility of two other consensus indicators are the focus of ongoing research. In the opinion of the panellists, treatment should be initiated in any male patients with classical FD aged at least 16 years, and in younger males with classical disease if early signs of organ damage appear. Female patients and male patients with non-classical disease should be treated based on existing guideline recommendations.

Detection of renal histological damage requires a biopsy, which is highly invasive, so the presence of other, less invasive early indicators could be sufficient grounds to start FD-specific treatment without biopsy data. The panel reached a consensus that early indicators of renal damage included microalbuminuria, glomerular hyperfiltration and podocyte inclusions in the presence of other renal lesions, such as signs of glomerulosclerosis or vasculopathy, which may occur even in patients without microalbuminuria (figure 2). ${ }^{22} 23$

Regarding cardiac indicators, consensus was reached on several early indicators of cardiac damage, including ECG 
A Renal indicators

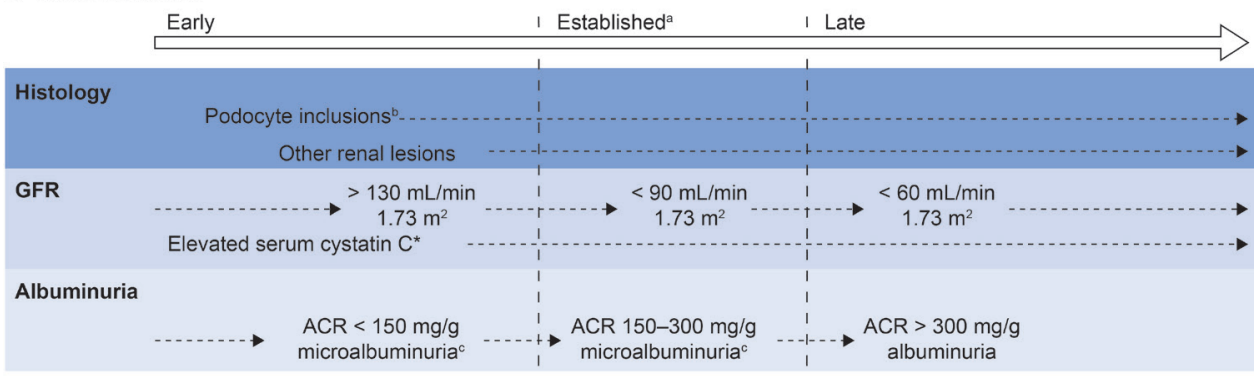

B Cardiac indicators

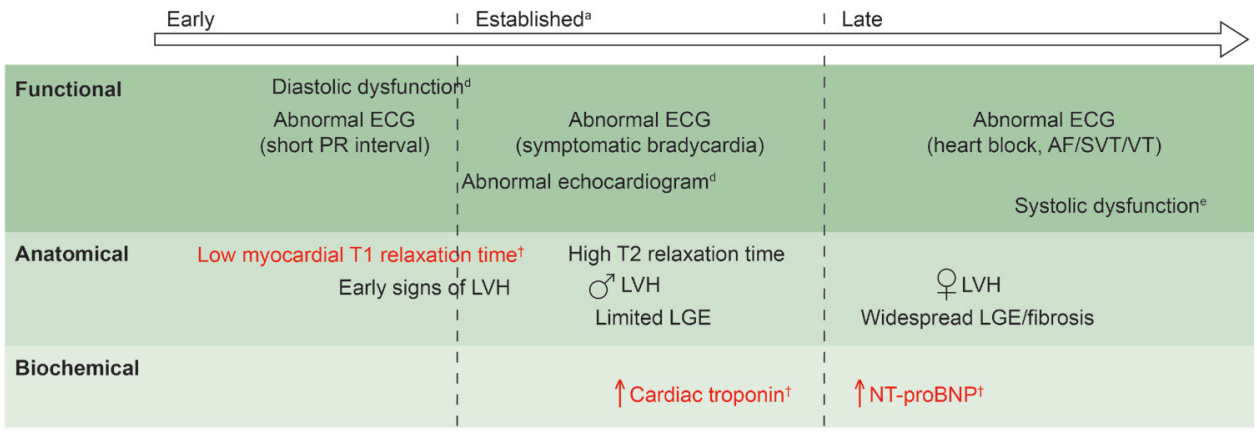

C Patient-reported/other indicators

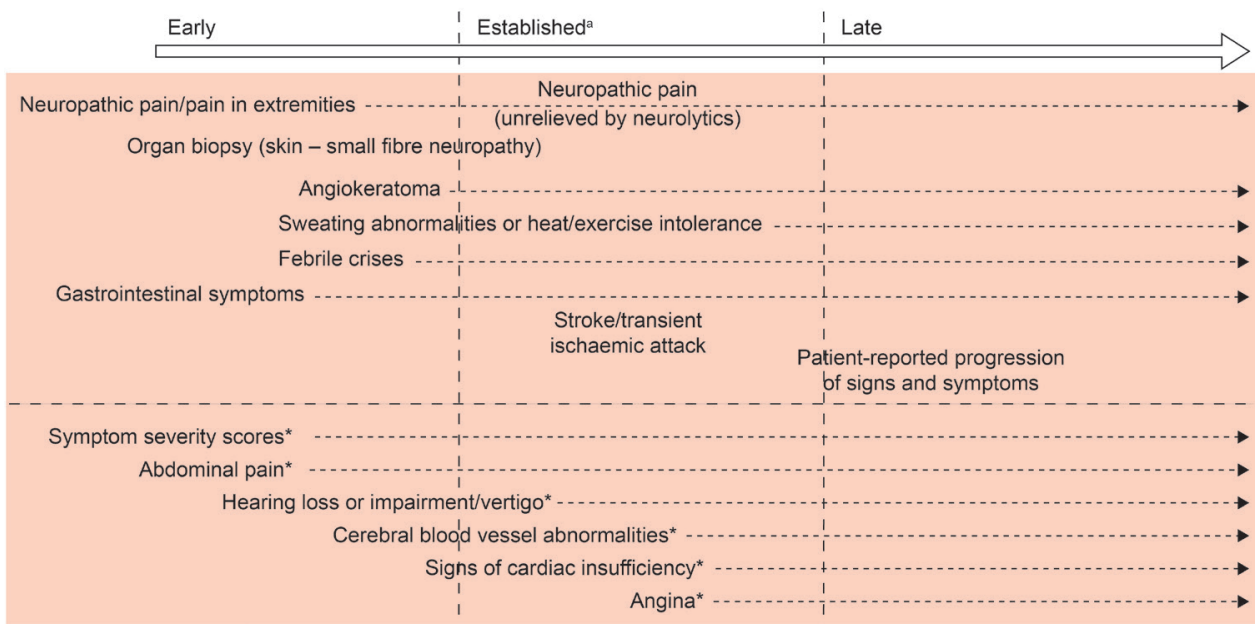

Figure 2 Chronology of consensus indicators. (A) *Indicator tested for, but not achieving, consensus in round 3. (B) †Indicators in red text achieved consensus both as currently used, and suitable for future adoption, because they are not available in all centres. Two further indicators (abnormal PET/MRI and increased serum lyso Gb3) that were included in round 2 of the initiative but were not taken forward to round 3 are not shown here based on guidance from the cochairs. (C) *Indicator tested for, but not achieving, consensus in round 3. Other indicators tested for, but not achieving, consensus, and which are not included here owing to their lack of specificity were: biomarkers; patient-reported outcomes; absenteeism owing to ill health; and palpitations. ${ }^{a}$ Indicators that currently would be likely to trigger FD-specific treatment initiation. ${ }^{\mathrm{b}} \mathrm{In}$ isolation, probably insufficient justification for FD-specific treatment initiation. ${ }^{\circ}$ Microalbuminuria could be a trigger for further investigation, such as confirmatory biopsy, and subsequent initiation of disease-specific treatment. Including decreased myocardial strain and strain rate, tissue Doppler abnormalities, enlarged left atrium, abnormal wall motion or pulmonary vein abnormalities. Including shortened PR interval, non-SVT and symptomatic bradycardia. ACR, albumin:creatinine ratio; AF, atrial fibrillation; FD, Fabry disease; GFR, glomerular filtration rate; LGE, late gadolinium enhancement; LVH, left ventricular hypertrophy; lyso Gb3, globotriaosylsphingosine; NT-proBNP, N-terminal probrain natriuretic peptide; PET, positron emission tomography; SVT, sustained VT; VT, ventricular tachycardia.

abnormalities (eg, shortened PR interval) elevated cardiac troponin, elevated NT-pro-BNP and low myocardial T1 relaxation times on cMRI, although the utility of the last may be limited by the low availability of $\mathrm{T} 1$ mapping by cMRI in specialist FD centres. Grade 1 diastolic dysfunction in early $\mathrm{FD}^{24}$ may be a useful indicator of cardiac changes, but perhaps only in young patients. Because LVH is an established sign of cardiac involvement in $\mathrm{FD}$, any tests revealing early stages of hypertrophy could be valuable in informing treatment decisions and could help to slow cardiac disease progression on treatment. ${ }^{25}$ Elevated high-sensitivity cardiac troponin and NT-pro-BNP levels are early signs of cardiac damage that might be detectable before signs that can be seen with cMRI. A concern 


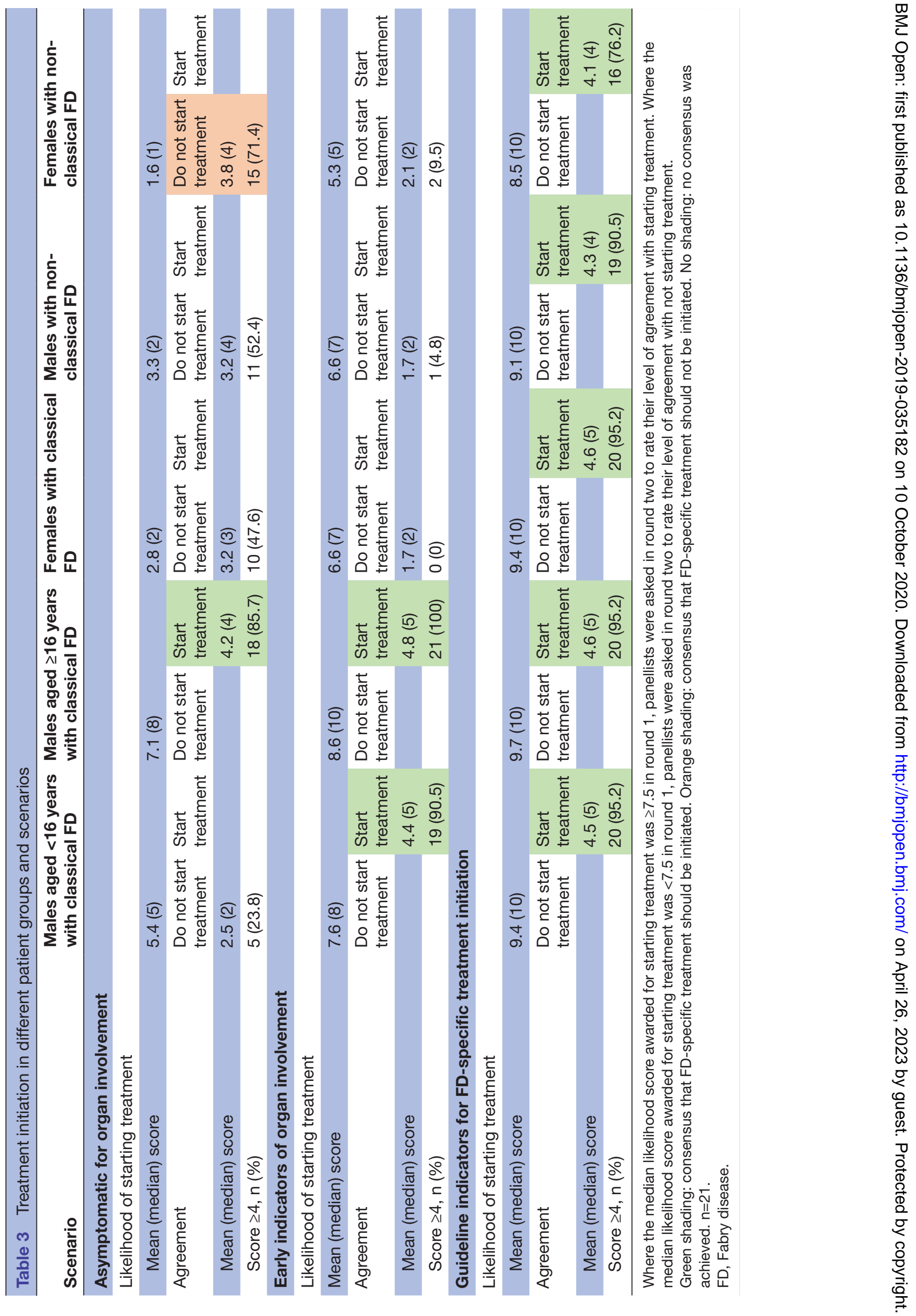




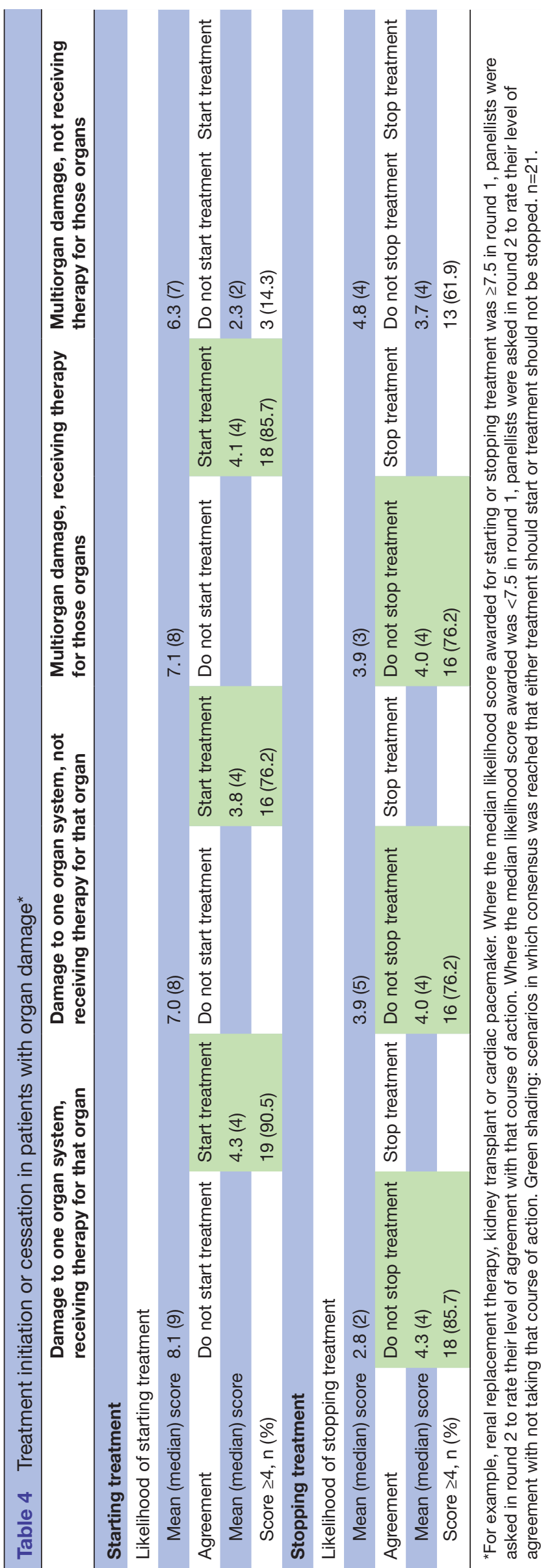

raised by panellists was that later manifestations of cardiac damage do not typically respond to FD-specific treatment. Histological markers have the potential to reveal very early cardiac tissue changes, but undertaking a cardiac biopsy is too invasive to be recommended as a routine screen for FD progression.

Other clinical and patient-reported early indicators of FD, such as neuropathic pain, gastroenterological symptoms and difficulties with hearing or balance, are wellknown signs and symptoms experienced by patients with FD. Such clinical features could contribute to a physician's decision to treat but may respond only partially to FD-specific treatment.

\section{Implications of the consensus indicators for the start of treatment}

The panel reached a consensus on initiating FD-specific treatment in predefined patient groups. In particular, the panel agreed that treatment should be initiated for all males $\geq 16$ years of age with the classical FD mutation regardless of symptom status. Similarly, the panel agreed that treatment should be initiated among males $<16$ years of age with classical FD demonstrating early or guidelineassociated indicators. However, there was no consensus on initiating treatment in asymptomatic males $<16$ years of age. In particular, consensus regarding early renal and cardiac indicators of disease progression could encourage FD centres to monitor for these indicators, pre-empting accrual of irreversible organ damage. Furthermore, agreement among the panel about the most suitable patient groups for FD-specific treatment initiation indicates that the current guideline recommendations ${ }^{1}$ could be updated, and the impact of early intervention could be audited for beneficial outcomes. Likewise, policymakers can use observational and longitudinal data to examine the cost-benefit implications of early treatment of patients for avoidable complications, as well as appropriate cessation of therapy in specific patient groups.

\section{Results of the PREDICT-FD initiative in context}

The PREDICT-FD modified Delphi initiative represents the broadest evaluation of early indicators of FD-specific treatment initiation to date. Previous Delphi initiatives have evaluated indicators specific to renal or cardiac organ damage, ${ }^{178}$ with a focus on tissue biopsy evaluation. However, biopsies are invasive and other approaches are available to aid early identification of disease progression. The use of biopsies in the diagnosis of FD was also key in a Delphi initiative exploring diagnosis, treatment and adverse event management. ${ }^{16}$ This Delphi panel reached conclusions similar to those of the PREDICT-FD panel regarding initiation of treatment. ${ }^{16}$ Both the cardiac and renal Delphi panels recognised serum lyso Gb3 levels as a potential indicator, although it might have limited specificity in kidney damage. ${ }^{1718}$ Lyso Gb3 has also been proposed as a potential primary biomarker for FD in other studies. ${ }^{26}{ }^{27}$ In the PREDICT-FD panel, there was no consensus on the use of lyso Gb3 as an early indicator of organ damage or treatment 
initiation, with the strongest marker of the importance of lyso Gb3 observed for cardiac damage.

\section{Strengths and weaknesses of the PREDICT-FD modified Delphi initiative}

The anonymised nature of Delphi methodology should minimise the possibility of bias often seen in face-to-face group interactions, thereby strengthening the validity of the consensus process. However, clinicians in a relatively small and highly specialised field may well be aware of the opinions of their peers, which may have influenced the responses provided in our study. With this qualification, the anonymity of the panellists was maintained until the Delphi stages were complete and the disease chronologies circulated for comment. Furthermore, the overall response rate was $>95 \%$, indicating that panellists' knowledge and opinions were well represented. However, because the importanceand agreement-rating steps in this Delphi consensus were opinion based, it is possible that a different consensus would have been reached, had the panel comprised different medical specialties. Thus, the generalisability of our findings is influenced by the panel composition and by the degree to which each panellist's perspective represents that of FD specialists not polled. Such shortcomings are implicit in the Delphi process and the findings require further evaluation in real-world clinical practice to confirm their relevance. Weaknesses of the methodology were the absence of a neutral response option for those unfamiliar with the relevance of an indicator during the importance rating stage, and that no controlled feedback was provided to panellists between rounds. Another was that no attempt was made to achieve consensus on the utility of indicators that did not meet the consensus criteria. Conceivably, this would have led to some indicators being completely discounted, leaving others whose utility remains to be proven.

\section{Conclusion and implications for future research}

The PREDICT-FD modified Delphi initiative achieved consensus on 27 early renal, cardiac, PNS, patient-reported and other indicators of disease progression that could prompt FD-specific treatment initiation earlier than is currently practised. These findings should raise awareness among physicians of the early clinical cues that should prompt consideration of disease-specific treatment initiation in FD, so that disease progression and irreversible organ damage in these patients is minimised or avoided. Empirically, early treatment is associated with better outcomes than delaying treatment of FD, but there is currently scant information about the responsiveness to treatment of many of the early indicators of disease progression identified in PREDICT-FD. Further evidence is needed to understand the latest stage at which treatment can be initiated to minimise the long-term complications of FD.

\footnotetext{
Author affiliations

${ }^{1}$ Lysosomal Storage Disorders Unit, Royal Free Hospital, London, UK ${ }^{2}$ Department of Haematology, University College London, London, UK ${ }^{3}$ Inborn Errors of Metabolism Reference Center, North Lisbon Hospital Center, Lisbon, Portugal
}

${ }^{4}$ Medicine Department, University of Lisbon, Lisbon, Portugal

${ }^{5}$ Lysosomal Disorders Unit, Addenbrooke's Hospital, Cambridge, UK

${ }^{6}$ Department of Medicine, University of Cambridge, Cambridge, UK

${ }^{7}$ Department and Laboratory of Paediatric Metabolic Disorders, Gazi University,

Ankara, Turkey

${ }^{8}$ Department of Cardiovascular, Respiratory, Nephrologic, Geriatric and

Anesthesiologic Sciences, University of Rome La Sapienza, Rome, Italy

${ }^{9}$ Department of Internal Medicine-Rheumatology, Croix Saint Simon Hospital, Paris, France

${ }^{10}$ Department of Cardiovascular Medicine, First Faculty of Medicine, Charles

University and General University Hospital, Prague, Czech Republic

${ }^{11}$ Cardiac Imaging Department, Barts Heart Centre, London, UK

${ }^{12}$ Department of Nephrology, Royal Melbourne Hospital, Parkville, Victoria, Australia

${ }^{13}$ Department of Medicine, The University of Melbourne - Parkville Campus, Melbourne, Victoria, Australia

${ }^{14}$ Department of Pediatrics, Taipei Veterans General Hospital, Taipei, Taiwan

${ }^{15}$ Institute of Clinical Medicine, National Yang-Ming University, Taipei, Taiwan

${ }^{16}$ Department of Endocrinology and Clinical Nutrition, University Hospital Zurich and

University of Zurich, Zurich, Switzerland

${ }^{17}$ Department of Internal Medicine, Psychiatry University Hospital Zurich, Zurich, Switzerland

${ }^{18}$ Department of Neurology, British Hospital of Buenos Aires, Buenos Aires, Argentina

${ }^{19}$ Instituto de Estudios Inmunológicos y Fisiopatológicos, UNLP - CONICET, La Plata, Argentina

${ }^{20}$ Institute of Metabolic Disease, Baylor Research Institute, Dallas, Texas, USA

${ }^{21}$ Department of Clinical Medicine, University of Bergen, Bergen, Norway

${ }^{22}$ Department of Medicine, Haukeland University Hospital, Bergen, Norway

${ }^{23}$ Department of Nephrology, Royal Perth Hospital, Perth, Western Australia, Australia

${ }^{24}$ Inherited Renal Diseases Unit, Autonomous University of Barcelona, Barcelona, Spain

${ }^{25}$ Department of Internal Medicine, General Hospital Slovenj Gradec, Slovenj Gradec, Slovenia

${ }^{26}$ Division of Nephrology, University of Alabama at Birmingham, Birmingham,

Alabama, USA

${ }^{27}$ Medicine, Dalhousie University, Halifax, Nova Scotia, Canada

${ }^{28}$ Fabry Support \& Information Group, Concordia, Missouri, USA

${ }^{29}$ Fabry International Network, Beveren, Belgium

${ }^{30}$ Oxford PharmaGenesis, Oxford, UK

${ }^{31}$ Division of Nephrology, Belcolle Hospital, Viterbo, Italy

Acknowledgements This initiative is endorsed by the Fabry International Network. The authors are grateful to 0xford PharmaGenesis, Oxford, UK, for providing administrative and project management support for the PREDICT-FD initiative and for medical writing, editorial support and project management support in preparation of this manuscript. This research and support from Oxford PharmaGenesis were funded by an unrestricted independent medical education grant (IME-GBR-15474) from Shire International (now part of Takeda Pharmaceutical Company), Zug, Switzerland. The authors chose independently to work with Oxford PharmaGenesis, without input from Shire International GmbH.

Contributors DAH and SF provided expert clinical insight throughout the development of the PREDICT-FD modified Delphi initiative, advised on the recruitment for the panel members and contributed to the concept, design and development of the initiative and the development of the questions for each round, as well as to the interpretation of the findings. MJR contributed to the design and development of the initiative and the development of the questions for each round, as well as to the interpretation of the findings. JJ provided expert guidance on the initiative design and questions for each round, and the interpretation of the findings. PA, PBD, FE, AF, OL, AL, J-CL, JCM, KN, D-MN, AN, UR, RR, PR, RS, ES, MT, RT, BV, DGW and MLW were voting members of the panel, and provided expert input at each round and on the interpretation of the findings. All authors contributed to the development and approval of the manuscript.

Funding Administration and coordination of the PREDICT-FD initiative and medical writing support were funded by an unrestricted independent educational grant (IME-GBR-15474) from Shire International GmbH, Zug, Switzerland, now part of Takeda Pharmaceutical Company. Shire/Takeda was not involved in the planning, design or delivery of the initiative. No reimbursement or funding was offered to the voting panel, to the cochairs or to any other organisation participating in the initiative. 
Competing interests DAH: advisory boards for Amicus, Sanofi, Shire (now part of Takeda); consulting fees from Amicus, Idorsia, Sanofi and Shire (now part of Takeda); honoraria from Amicus, Sanofi and Shire (now part of Takeda). PA: research grant and honoraria from Shire (now part of Takeda); honoraria from Amicus, Biomarin, Sanofi and Ultragenyx. PBD: speaker honoraria from and advisory boards for Takeda and Sanofi; consultancy for Sanofi. FE: travel grants and speaker honoraria from Pfizer, Sanofi Genzyme and Takeda. AF: research grants from Amicus and Shire. OL: travel grants and speaker honoraria from Amicus, Sanofi Genzyme and Shire HGT. AL: speaker's honoraria or consultation fees from Amicus, Sanofi Genzyme and Takeda. J-CL: speaker's honoraria and consultation fees from Shire. JCM: research grant and speaker honoraria from Sanofi Genzyme; advisory board for, and honoraria from ST; consulting fees from 4DMT. KN: research support and/or honoraria from Amicus, Idorsia, Protalix, Sanofi and Shire (now part of Takeda); advisory boards for Amicus, Sanofi and Shire (now part of Takeda). D-MN: research funding from Shire (now part of Takeda) and Sanofi. AN: honoraria and research support from Sanofi Genzyme and Shire (now part of Takeda). UR: advisory boards for Amicus, Chiesi, Idorsia and Shire; travel grants and honoraria from Amicus, Genzyme and Shire. RR: travel grants and speaker honoraria from Amicus and Shire HGT (now part of Takeda). PR: advisory board, consulting fees and research grant from Shire (now part of Takeda). RS: advisory boards for Amicus, Sanofi, Shire (now part of Takeda); honoraria from Amicus and Shire; research funding from Idorisia, Protalix, Sanofi Genzyme and Takeda. ES: speaker's fees and travel support from Amicus, Sanofi Genzyme and Shire; advisory board honoraria from Amicus and Sanofi Genzyme. MT: advisory boards for Amicus, Sanofi and Shire (now part of Takeda); travel grants and speaker's honoraria from Amicus, Sanofi and Shire; research funding from Amicus, AVROBIO and Idorsia. RT: travel grants, speaker's honoraria or consultation fees from Amicus, Sanofi Genzyme and Takeda. BV: speaker's fees and travel support from Greenovation, Sanofi Genzyme and Shire/Takeda; advisory board honoraria from Sanofi Genzyme. DGW: advisory boards for Amicus, Avrobio, Freeline Therapeutics, 4D-MT Technology, Idorsia and Protalix; honoraria and travel expenses from Amicus, Protalix and Sanofi; and equity interest in Reata Pharmaceuticals. MLW: advisory boards for Amicus, Sanofi and Shire (now part of Takeda); honoraria from Amicus, Sanofi and Shire; research funding from Amicus, Idorisia, Protalix and Shire. JJ: honoraria from Sanofi; travel expenses from Amicus and Sanofi. MJR is an employee of Oxford PharmaGenesis (Oxford, UK). SF: advisory boards for Amicus; consulting fees from Shire (now part of Takeda); contracted research from Shire (now part of Takeda); honoraria from Amicus, Sanofi and Shire (now part of Takeda); speaker's bureau for Amicus, Sanofi and Shire (now part of Takeda); travel expenses from Amicus, Sanofi and Shire (now part of Takeda).

Patient and public involvement Patients and/or the public were involved in the design, or conduct, or reporting, or dissemination plans of this research. Refer to the Methods section for further details.

Patient consent for publication Not required.

Ethics approval No patient-level data were used in this study and no ethical approval was sought.

Provenance and peer review Not commissioned; externally peer reviewed.

Data availability statement No data are available. All key data for this study are included in this article or uploaded as online supplementary information.

Open access This is an open access article distributed in accordance with the Creative Commons Attribution Non Commercial (CC BY-NC 4.0) license, which permits others to distribute, remix, adapt, build upon this work non-commercially, and license their derivative works on different terms, provided the original work is properly cited, appropriate credit is given, any changes made indicated, and the use is non-commercial. See: http://creativecommons.org/licenses/by-nc/4.0/.

\section{ORCID iDs}

Andrea Frustaci http://orcid.org/0000-0002-5748-0725

Mark J Rolfe http://orcid.org/0000-0002-9248-1563

\section{REFERENCES}

1 Biegstraaten M, Arngrímsson R, Barbey F, et al. Recommendations for initiation and cessation of enzyme replacement therapy in patients with Fabry disease: the European Fabry working group consensus document. Orphanet J Rare Dis 2015;10:36.

2 Rombach SM, Hollak CEM, Linthorst GE, et al. Cost-effectiveness of enzyme replacement therapy for fabry disease. Orphanet $J$ Rare Dis 2013;8:29.
3 Hughes DA, Mlilligan A, Mehta A. Home therapy for lysosomal storage disorders. Br J Nurs 2007;16:1384-9.

4 Milligan A, Hughes D, Goodwin S, et al. Intravenous enzyme replacement therapy: better in home or hospital? Br J Nurs 2006;15:330-3.

5 Germain DP, Charrow J, Desnick RJ, et al. Ten-year outcome of enzyme replacement therapy with agalsidase beta in patients with Fabry disease. J Med Genet 2015;52:353-8.

6 Hopkin RJ, Cabrera G, Charrow J, et al. Risk factors for severe clinical events in male and female patients with Fabry disease treated with agalsidase beta enzyme replacement therapy: data from the fabry registry. Mol Genet Metab 2016;119:151-9.

7 Weidemann F, Niemann M, Sommer C, et al. Interdisciplinary approach towards female patients with Fabry disease. Eur J Clin Invest 2012;42:455-62.

8 Hopkin RJ, Jefferies JL, Laney DA, et al. The management and treatment of children with Fabry disease: a United States-based perspective. Mol Genet Metab 2016;117:104-13.

9 Talbot AS, Lewis NT, Nicholls KM. Cardiovascular outcomes in Fabry disease are linked to severity of chronic kidney disease. Heart 2015;101:287-93.

$10 \mathrm{Kim} \mathrm{JH}$, Lee BH, Hyang Cho J, et al. Long-term enzyme replacement therapy for Fabry disease: efficacy and unmet needs in cardiac and renal outcomes. J Hum Genet 2016;61:923-9.

11 Arends M, Biegstraaten M, Hughes DA, et al. Retrospective study of long-term outcomes of enzyme replacement therapy in Fabry disease: analysis of prognostic factors. PLoS One 2017;12:e0182379.

12 Lenders M, Schmitz B, Stypmann J, et al. Renal function predicts long-term outcome on enzyme replacement therapy in patients with Fabry disease. Nephrol Dial Transplant 2017;32:2090-7.

13 Arends M, Wijburg FA, Wanner C, et al. Favourable effect of early versus late start of enzyme replacement therapy on plasma globotriaosylsphingosine levels in men with classical Fabry disease. Mol Genet Metab 2017:121:157-61.

14 Kozor R, Grieve SM, Tchan MC, et al. Cardiac involvement in genotype-positive Fabry disease patients assessed by cardiovascular MR. Heart 2016;102:298-302.

15 Imbriaco M, Pellegrino T, Piscopo V, et al. Cardiac sympathetic neuronal damage precedes myocardial fibrosis in patients with Anderson-Fabry disease. Eur J Nucl Med Mol Imaging 2017;44:2266-73.

16 Concolino D, Degennaro E, Parini R, et al. Delphi consensus on the current clinical and therapeutic knowledge on Anderson-Fabry disease. Eur J Intern Med 2014;25:751-6.

17 Smid BE, van der Tol L, Cecchi F, et al. Uncertain diagnosis of Fabry disease: consensus recommendation on diagnosis in adults with left ventricular hypertrophy and genetic variants of unknown significance. Int J Cardiol 2014;177:400-8.

18 van der Tol L, Svarstad E, Ortiz A, et al. Chronic kidney disease and an uncertain diagnosis of Fabry disease: approach to a correct diagnosis. Mol Genet Metab 2015;114:242-7.

19 Hsu CC, Sandford BA. The Delphi technique: making sense of consensus. Pract Assess Res Eval 2007;12:1-8.

20 Bousquet J, Winchester C, Papi A, et al. Inhaled corticosteroid/ long-acting $\beta_{2}$-agonist combination therapy for asthma: attitudes of specialists in Europe. Int Arch Allergy Immunol 2012;157:303-10.

21 Mehta A, Kuter DJ, Salek SS, et al. Presenting signs and patient co-variables in Gaucher disease: outcome of the Gaucher earlier diagnosis consensus (GED-C) Delphi initiative. Intern Med $J$ 2019;49:578-91.

22 Tøndel C, Bostad L, Hirth A, et al. Renal biopsy findings in children and adolescents with Fabry disease and minimal albuminuria. $A m \mathrm{~J}$ Kidney Dis 2008:51:767-76.

23 Ramaswami U, Bichet DG, Clarke LA, et al. Low-dose agalsidase beta treatment in male pediatric patients with Fabry disease: a 5-year randomized controlled trial. Mol Genet Metab 2019;127:86-94.

24 Weidemann F, Beer M, Kralewski M, et al. Early detection of organ involvement in Fabry disease by biomarker assessment in conjunction with LGE cardiac MRI: results from the SOPHIA study. Mol Genet Metab 2019;126:169-82.

25 Goker-Alpan O, Longo N, McDonald M, et al. An open-label clinical trial of agalsidase alfa enzyme replacement therapy in children with Fabry disease who are naïve to enzyme replacement therapy. Drug Des Devel Ther 2016;10:1771-81.

26 Nowak A, Mechtler TP, Desnick RJ, et al. Plasma LysoGb3: a useful biomarker for the diagnosis and treatment of Fabry disease heterozygotes. Mol Genet Metab 2017:120:57-61.

27 Maruyama H, Miyata K, Mikame M, et al. Effectiveness of plasma lyso-Gb3 as a biomarker for selecting high-risk patients with Fabry disease from multispecialty clinics for genetic analysis. Genet Med 2019;21:44-52. 